\title{
Optimal Calibration Method for Water Distribution Water Quality Model
}

QUERY SHEET 


\section{Optimal Calibration Method for Water Distribution Water ${ }_{3}$ Quality Model}

4 Zheng Yi Wu

5 Bentley Systems, Incorporated, Haestad Methods Solution Center, Watertown, 6 CT, USA

7 A water quality model is to predict water quality transport and fate throughout a 8 water distribution system. The model is not only a promising alternative for analyzing 9 disinfectant residuals in a cost-effective manner, but also a means of providing 10 enormous engineering insights into the characteristics of water quality variation and 11 constituent reactions. However, a water quality model is a reliable tool only if it predicts what a real system behaves. This paper presents a methodology that enables a modeler to efficiently calibrate a water quality model such that the field observed water quality values match with the model simulated values. The method is formulated to adjust the global water quality parameters and also the element-dependent water quality reaction rates for pipelines and tank storages. A genetic algorithm is applied to optimize the model parameters by minimizing the difference between the model-predicted values and the field-observed values. It is seamLessly integrated with a well-developed hydraulic and water quality modeling system. The approach has provided a generic tool and methodology for engineers to construct the sound water quality model in expedient manner. The method is applied to a real water system and demonstrated that a water quality model can be optimized for managing adequate water supply to public communities.

Key Words: Water distribution; Water supply; Water quality model; Chlorine decay;

Simulation; Calibration; Optimization; Genetic algorithm.

\section{INTRODUCTION}

Drinking water quality is essential to public heath. Although water treatment is a common practice for supplying good quality of water from a source, maintaining an adequate water quality throughout a distribution system is a daunting task. The challenges remain in the complex pipe geometry,

Address correspondence to Zheng Yi Wu, Bentley Systems Inc., Haestad Methods Solution Center, 27 Siemon Company Drive, Suite 200W, Watertown CT06795, USA; E-mail: Zheng.Wu@bentley.com 
sophisticated network connectivity, various system operation controls, temporal and spatial variation of water demand, and also intriguing constituent reaction in bulk water and in between the water and pipe walls. Sampling and continuously monitoring water quality at appropriate locations have played an important role to minimize the risk of inadequate water quality to public health, however, but sampling only presents a limited picture of water quality in that there are only a few monitoring points and monitoring cannot be used to predict future conditions or perform "what if" analyses due to the limited coverage and the high cost. Thus, using a welldeveloped hydraulic and water quality model is an important approach for simulating the hydraulic and water quality dynamics for all elements in a system.

Water quality modeling has become an increasingly common practice for water utilities around world. It is formulated as a mathematical model and developed as computer-based tool to predict water quality transport and fate within a water distribution system according to the network flow dynamics. The model is not only a promising technology for predicting disinfectant residuals in a cost-effective manner, but also a means of providing enormous engineering understanding in the dynamics of water quality variation and the sophisticated process of constituent reactions that occur in water distribution systems. The early development of water quality models was based upon the steady-state hydraulic simulation of mass conservation law. The models ${ }^{[1-4]}$ determined the water quality spatial distribution of a constituent throughout a pipeline network under static hydraulic conditions.

Although the steady-state water quality model proved to be useful for investigating the overall movement of a contaminant under constant conditions, the system hydraulics is constantly changing in tank levels, valve settings, pump operating status and nodal demand. Therefore, the need for the models that represent the dynamics of contaminant movement led to the development of better water quality models under temporally varying conditions. Dynamic models of water quality in distribution systems explicitly take into account of changing in flows through pipelines and storage facilities over an extended period of time. A number of solution methods ${ }^{[5-9]}$ are developed for dynamic water quality models. They can be classified spatially as either Eulerian or Lagrangian and temporally as time-driven or event-driven methods. Each of these methods assumes that a hydraulic model determines the flow direction and velocity in each pipe at specific time intervals over an extended period. Within each interval, referred as hydraulic time step, the pipe flow velocity remains constant, the simulation of a constituent movement and reaction proceeds in a smaller time step (so-called water quality time step). Thus the dynamic approach is more realistic than steady-state methods in simulating systematic condition of water quality transportation and reaction in distribution networks. 
However, a water quality model is an effective and reliable analysis tool only when the constituent reaction and decay/growth mechanisms are properly defined. This can only be achieved by calibrating the water quality model using the field observed and lab-tested water quality data. Like hydraulic model calibration, water quality model calibration is a time-consuming and tedious process by manually adjusting model parameters. In this article, a competent genetic algorithm-based calibration approach for calibrating a water quality model is presented. It provides modelers a flexible optimization modeling tool to facilitate the water quality model calibration task. In order to develop an effective calibration method, it is important to understand the insights into water quality model formulation. A brief overview of water quality model is given next.

\section{WATER QUALITY MODEL}

Water quality model for a water distribution system is based upon Reynolds transport theorem (RTT) and formulated for one-dimensional, unsteady small fluid parcel as follows.

$$
\frac{\partial C}{\partial t}+V \frac{\partial C}{\partial x}=R(C)
$$

where $C$ is the concentration of a constituent; $t$ is time; $V$ is the flow velocity; $x$ is the distance and $R$ represents the constituent reaction relationship. Water quality model for a water distribution system used in this paper is based upon a parcel tracking algorithm. ${ }^{[8,9]}$ It tracks the change in water quality of discrete parcels of water as they move along pipes and mixes together at junctions between fixed-length time steps. During a simulation, the water quality in each parcel is updated to reflect any reaction that may have occurred over the time step. The water from the leading parcels of pipes with flow into each junction is blended together, along with any external inflow to the junction, to compute a new water quality value at the junction. The volume contributed from each parcel equals the product of its pipe's flow rate and the time step. If this volume exceeds that of the leading parcel then the leading parcel is destroyed and the next parcel in line behind it begins to contribute its volume. New parcels are created in pipes with flow out of each junction. The parcel volume equals the product of the pipe flow and the time step.

To reduce on the number of parcels, a new parcel is formed if the new junction quality differs by a user-specified tolerance from that of the last parcel in the outflow pipe. If the difference in quality is below the tolerance then the size of the last parcel is simply increased by the volume of flow released into the pipe over the time step with no change in quality. Initially each pipe in the network consists of a single parcel whose quality equals the initial quality assigned to the upstream node. The water quality simulation tracks the growth 
or decay of a substance by reaction as it travels through a distribution system. To do this, it needs to know the rate at which the substance reacts and how this rate might depend on substance concentration. Reactions can occur both within the bulk flow and with material along the pipe wall. Bulk fluid reactions can also occur within tanks.

\section{Bulk Reaction}

Bulk flow reactions are the reactions that occur in the main flow stream of a pipe or in a storage tank, unaffected by any processes that might involve the pipe wall. A water quality model simulates these reactions using $n$-th order kinetics, where the instantaneous rate of reaction ( $R$ in unit of mass/volume/time) is assumed to be concentration-dependent, given as:

$$
R(C)=K_{b} C^{n}
$$

where $K_{b}$ is a bulk rate coefficient; $C$ is reactant concentration (mass/volume) and $n$ is a reaction order. $K_{b}$ has units of concentration raised to the $(1-n)$ power divided by time. It is positive for growth reactions and negative for decay reactions. It also considers reactions where a limiting concentration exists on the ultimate growth or loss of the substance. In this case the rate expression for a growth reaction becomes

$$
R(C)=K_{b}\left(C_{L}-C\right) C^{(n-1)}
$$

where $C_{L}=$ the limiting concentration. For decay reactions $\left(C_{L}-C\right)$ is replaced by $\left(C-C_{L}\right)$.

Thus, there are three parameters $\left(K_{b}, C_{L}\right.$, and $\left.n\right)$ that are used to characterize bulk reaction rates. Different values of these parameters lead to different kinetic models. They need to be carefully calibrated for the pipes and tanks in a water distribution system.

\section{Wall Reaction}

In addition to bulk flow reactions, constituent reactions occur with material on or near the pipe wall. The rate of this reaction is dependent on the concentration in the bulk flow and pipe wall conditions, given as:

$$
R(C)=(A / V) K_{w} C^{n}
$$

Where $K_{w}$ is a wall reaction rate coefficient and $(A / V)$ is the surface area per unit volume within a pipe. It converts the mass reacting per unit of wall area to a per unit volume basis. $n$ is the wall reaction order taking value of either 0 or 1 , so that the unit of $K_{w}$ is either mass/area/time or length/time. Both $K_{w}$ and $n$ are site specific and need to be calibrated for water distribution pipes. 


\section{CALIBRATION FORMULATION}

149 To calibrate a water quality model for analyzing any constituent (not just Chlo150 rine decay), it is important to adjust the parameters that govern the reaction mechanism. It includes bulk reaction and pipe wall reaction parameters.

\section{Bulk Water Reaction Calibration}

Bulk reaction rate is conventionally obtained by conducting bottle test in a laboratory, namely taking bottles of sample water and measuring the constituent concentration of the bottle water over time test to determine the bulk water reaction rate. For chlorine decay, one can measures the residual of chlorine over time, so that bulk reaction/decay can be gauged by the bottle test. Bottle test is recommended for determining the bulk reaction coefficient such as chlorine decay factor. It provides a good baseline value and reference for constructing a water quality model. Although bulk reaction coefficient can be attained by bottle test, real bulk reaction may vary from one portion of a system to another due to dynamic flow conditions and mixing of multiple water sources. The real bulk reaction mechanism needs to be calibrated throughout a distribution system. Bulk water reaction is generally characterized by three parameters including:

(i) Bulk reaction coefficient $K_{b}$;

(ii) Bulk reaction order $n_{b}$;

(iii) Concentration limit $C_{L}$.

Bulk reaction parameters need to be adjusted for both pipe and tank elements. The pipes that are of the similar characteristics are allowed to be grouped into one calibration group for bulk reaction coefficient adjustment. The bulk reaction groups are set up in a similar fashion to the roughness group, ${ }^{[10]}$ prescribed with minimum, maximum values and an increment for each pipe group and tank. The same reaction parameters are applied to the pipes in one calibration group. This reduces the number of the calibration parameters. Tank bulk reaction coefficient is calibrated individually for each storage facility. By adjusting all the parameters $\left(K_{b}, n_{b}\right.$ and $\left.C_{L}\right)$, a water quality model can be calibrated to simulate the bulk water reaction of not only chlorine decay, but also the other reaction mechanisms such as firstorder saturation growth, two-component second-order growth, two-component second-order decay and the other reaction mechanisms.

\section{Pipe Wall Reaction Calibration}

Pipe wall reaction is characterized by the wall coefficient $\left(K_{w}\right)$ and reaction order $\left(n_{w}\right)$. Both parameters are closely related to pipe material and pipe wall 
physical conditions such as encrustation and tuburculation of corrosion products. Two methods are developed for calibrating pipe wall reaction mechanism.

\section{Direct Calibration}

Direct calibration is to directly optimize the pipe wall reaction coefficient and reaction order for a group of pipes. Since the wall reaction mechanism is expected to have the same behavior for the pipes of the same characteristics (age, material and location). Similar to the roughness calibration group, ${ }^{[11]}$ pipes of the same characteristics are allowed to be aggregated and treated as a set of common calibration parameters, wall coefficient and order are calibrated between the minimum and maximum values with an increment specified by a modeler.

\section{Correlation Calibration}

Alternatively, pipe wall reaction can be calibrated by adjusting a correlation factor. It is well known that as metal pipes age their roughness tends to increase due to encrustation and tuburculation of corrosion products on the pipe walls. This increase in roughness produces a lower Hazen-Williams $C$-factor or a higher Darcy-Weisbach roughness coefficient, resulting in greater frictional headloss in flow through the pipe. There is some evidence ${ }^{[12]}$ to suggest that the same processes that increase a pipe's roughness with age also tend to increase the reactivity of its wall with some chemical species, particularly chlorine and other disinfectants. Each pipe's wall reaction coefficient $\left(K_{w}\right)$ can be a function of the coefficient used to describe its roughness. A different function applies depending on the formula used to compute headloss through a pipe:

$$
\begin{array}{ll}
\text { Hazen-Williams: } & K_{w}=F / C \\
\text { Darcy-Weisbach: } & K_{w}=-F / \log (e / d) \\
\text { Chezy-Manning: } & K_{w}=F^{*} N
\end{array}
$$

where $C$ is Hazen-Williams $C$-factor; $e$ is Darcy-Weisbach roughness, $d$ is pipe diameter, $N$ is Manning roughness coefficient and $F$ is the coefficient of correlation of wall reaction and pipe roughness. The coefficient $F$ must be developed from site-specific field measurements and will have a different meaning depending on which headloss equation is used. The advantage of using this approach is that it requires only a single parameter $F$, to allow wall reaction coefficients to vary throughout the network in a physically meaningful way. This is because a hydraulic model must be calibrated before undertaking a water quality model calibration. Therefore, pipe roughness should be a known value for water quality model calibration. In this case, modelers may choose to 
just calibrate the correlation factor for Chlorine pipe wall reaction mechanism. Correlation factor adjustment can also be conducted for a group of pipes or globally for an entire system.

One calibration solution represents one set of parameters that define the bulk water reaction and pipe wall reaction mechanism. Each possible solution is passed to a hydraulic and water quality model which produces the simulation results of water quality concentrations in a system. The simulated concentration values are compared with the observed values. The comparison is quantified as a goodness-of-fit between the simulated and the observed values. The goodness-of-fit is defined as a fitness or calibration objective function in the following section.

\section{Calibration Objectives}

The objective of water quality model calibration is to minimize the difference between the field observed and the model simulated constituent concentrations. Assume the field observed concentration be represented by $C_{i}{ }^{o b s}\left(t_{j}\right)$ at time $t_{j}$ for monitoring location $i$ and collected over $N$ time steps at $M$ locations while the model simulated concentration is noted as $C_{i}{ }^{\operatorname{sim}}\left(t_{j}\right)$. The calibration objective can be measured in many different ways formulated as follows.

Minimize difference square:

$$
\text { Fitness }=\frac{\sum_{i=1}^{M} \sum_{j=1}^{N}\left(C_{i}^{o b s}\left(t_{j}\right)-C_{i}^{s i m}\left(t_{j}\right)\right)^{2}}{N \times M}
$$

Minimize absolute difference:

$$
\text { Fitness }=\frac{\sum_{i=1}^{N} \sum_{j=1}^{M}\left|C_{i}^{\text {obs }}\left(t_{j}\right)-C_{i}^{\text {sim }}\left(t_{j}\right)\right|}{N \times M}
$$

Minimize absolute maximum difference:

$$
\text { Fitness }=\max _{i, j}\left|C_{i}^{\text {obs }}\left(t_{j}\right)-C_{i}^{\text {sim }}\left(t_{j}\right)\right|
$$

Minimize sum of absolute mean difference:

$$
\text { Fitness }=\sum_{i=1}^{M} \frac{\sum_{j=1}^{N}\left|C_{i}^{o b s}\left(t_{j}\right)-C_{i}^{\text {sim }}\left(t_{j}\right)\right|}{N}
$$

When chemical concentration is collected at a sampling/monitoring station, it may not be measured at a regular time step. To compare between the observed and simulated concentration, the simulated result must be obtained for the same time as the observed value is collected. When the simulation time step 
does not exactly match the time step of data collection, the simulated concentration is attained by interpolating the results at two adjacent computation time steps for the same monitor location/node. The coefficients for bulk water and pipe wall reaction can be calibrated for pipe groups while the reaction orders and concentration limit are global parameters for a system.

Water quality calibration, formulated as above, is a nonlinear implicit optimization problem. It is solved by using the same methodology for hydraulic model calibration by Wu et al. ${ }^{[10,11]}$ In fact, the hydraulic calibration has been extended to include the calibration of water quality parameters by means of the competent genetic algorithm. ${ }^{[13]}$

\section{SOLUTION METHODOLOGY}

The implementation of water quality calibration algorithm is illustrated in Figure 1. The information flows in both directions between the end-user and the data storage and thus enables engineers to effectively manage the data and calibrate a model by exploiting the powerful combination of GA optimizer and hydraulic network simulator, both are embodied into one modeling system. It consists of a user interface, calibration evaluation module, GA optimizer, hydraulic and water quality simulation model.

A user interface on a personal computer or other workstation lends the user the ability to enter the field observed data, select the representative demand loading, corresponding boundary conditions (including pump operating status, valve settings and tank levels) and calibration criteria. It enables a modeler to intuitively set up calibration, persistently conducting calibration tasks and graphically presenting results.

An initial calibration model is established by performing the extended period hydraulic simulation. The results are saved in the file that is repetitively used for water quality analysis of each calibration solution. A calibration run may proceed by either interactively adjusting calibration parameters (manually set a value for each parameter), that is to bypass the genetic algorithm optimizer, or presenting the data to GA optimizer to automatically search for the optimal and near optimal calibration solutions. Without activating the GA optimizer, the user-estimated model parameters are submitted to the hydraulic and water quality simulator. It predicts the water quality responses that are passed back to calibration evaluation module. The goodness-of-fit is calculated and reported to a user. Modelers can estimate the parameters and iterate over the process to enhance model calibration. In contrast, calibration can proceed with GA optimizer searching for the optimal solution. The GA optimizer will automatically generate and optimize the calibration solutions. Each trial solution, along with the selected data sets, corresponding loading and boundary conditions, is submitted to hydraulic network simulator for 


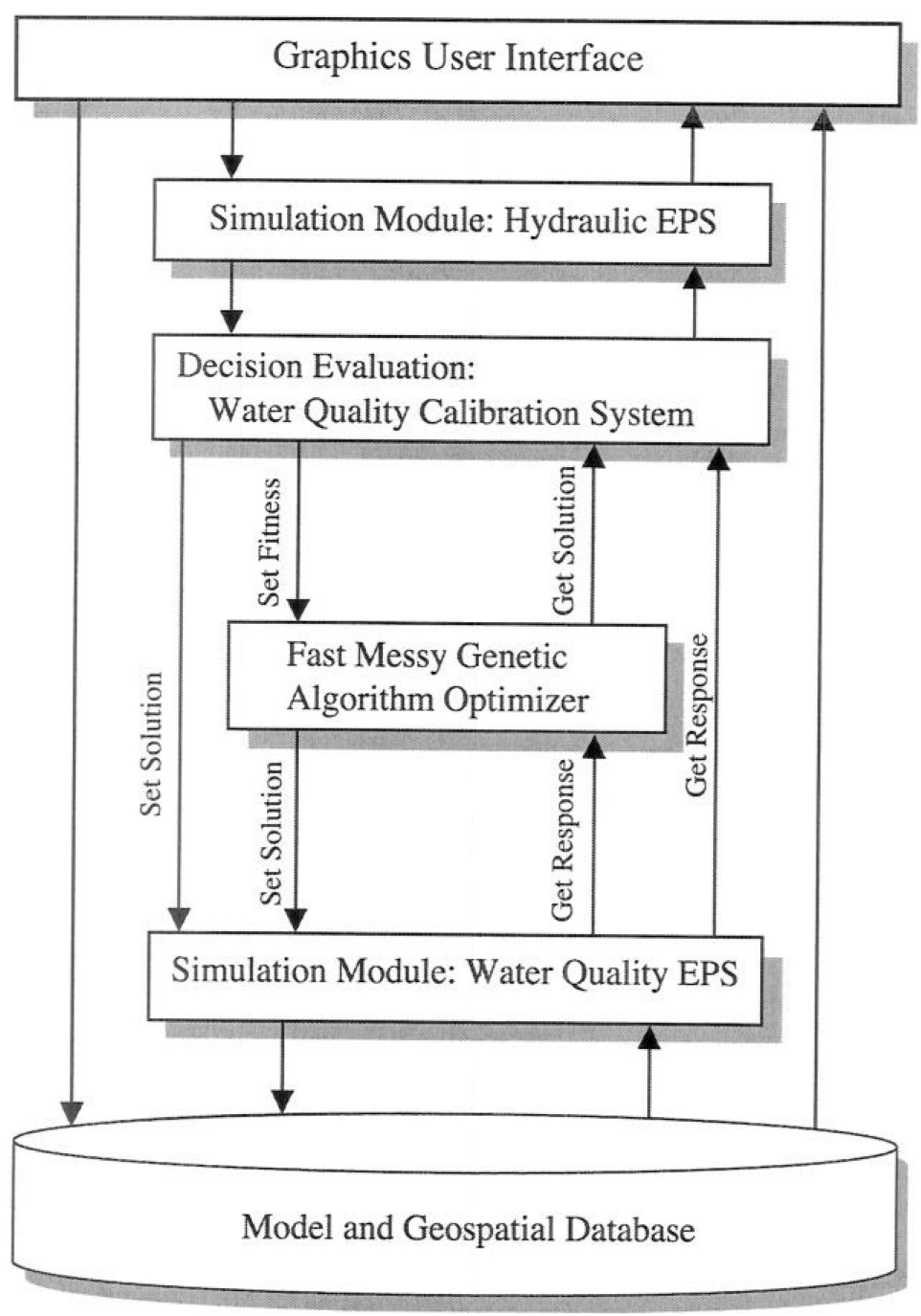

Figure 1: Darwin water quality calibration framework and data flow.

predicting the water quality results. The model simulated results are passed back to the calibration evaluation module and used to calculate the fitness for the solution. It enables the GA-optimizer to evolve the calibration solutions.

In addition, all the input data including the field data, calibration results and calibration run settings including the number of top solutions to be 296 reported, optimization stop criteria and GA parameters are consistently per297 sisted in a modeling database along with the simulation model. This permits 298 engineers to revisit the calibration process any time. It also helps modelers to better manage a project over time. The integrated implementation provides the 
10

300

301

302

303

304

305

306

307

308

309

310

311

312

313

314

315

316

317

318

319

320

321

322

323

324

325

326

327

328

329

330

331

332

333

334

335

336

337

338

339

Wu

powerful features of hydraulic and water quality network modeling paradigm. It has been applied to the optimization of the water quality model for chlorine decay study by Vasconcelos et al. ${ }^{[12]}$

\section{CASE STUDY}

Oberlin system, as shown as in Figure 2, has been demonstrated as an example of applying the calibration method to construct an accurate water quality model. Oberlin zone is a relatively isolated system receiving water from one source. It is a portion of Harrisburg water system within United Water service area. There is no storage facility in the zone. The isolated Oberlin zone was used for the project of chlorine decay kinetics study ${ }^{[12]}$ funded by America Water Works Research Fund (AWWARF). Chlorine concentration data were collected at 27 locations over 35 hours for capturing the balance of water quality state. This field data set provides an excellent example for water quality calibration. Both bulk water and pipe wall reaction parameters are calibrated to minimize the difference between the observed and simulated chlorine concentrations.

For chlorine reaction coefficients (bulk water and pipe wall) are adjusted for pipe groups. All the pipes are grouped into three groups. For each group, pipe wall correlation factor is specified within a range of -1000.0 and -100.0 with increment of 5.0. Bulk reaction coefficient is set up within a range of -2.0 and -0.1 in unit of $(\mathrm{mg} / \mathrm{L})^{(1.0-\mathrm{n}) / \mathrm{s}}$ with increment of 0.05 . Reaction orders are treated as global model parameter, so that they are adjusted for the entire zone. Bulk reaction order is between 1 and 5 while pipe wall reaction order can be either 0 or 1 .

Any of the four fitness functions can be selected to run water quality calibration. However, to compare the results with the previous study, fitness type of minimizing the sum of absolute mean differences is applied to this case study, namely, the fitness of calibration solution is calculated as the sum of absolute mean differences between the observed mean and the simulated mean over 35 hours for all the 27 locations. The Darwin Calibrator run is performed to optimize the water quality parameters. The comparison is presented, as in Figure 3, for both free chlorine decay and the calibrated average absolute errors of all monitor locations. The results obtained illustrate that it effectively improved the goodness-of-fit between the observed and predicted water quality when comparing to the free chlorine decay (all the points would fall on a straight line if agreement was perfect). Without the calibration on water quality model, the field observed water quality near the source appears to match well with the simulated results as shown in Figure 4. This is due to the fact that there is not much reaction/decay has occurred in the bulk water, and between the pipe wall and water right after chlorine is put 


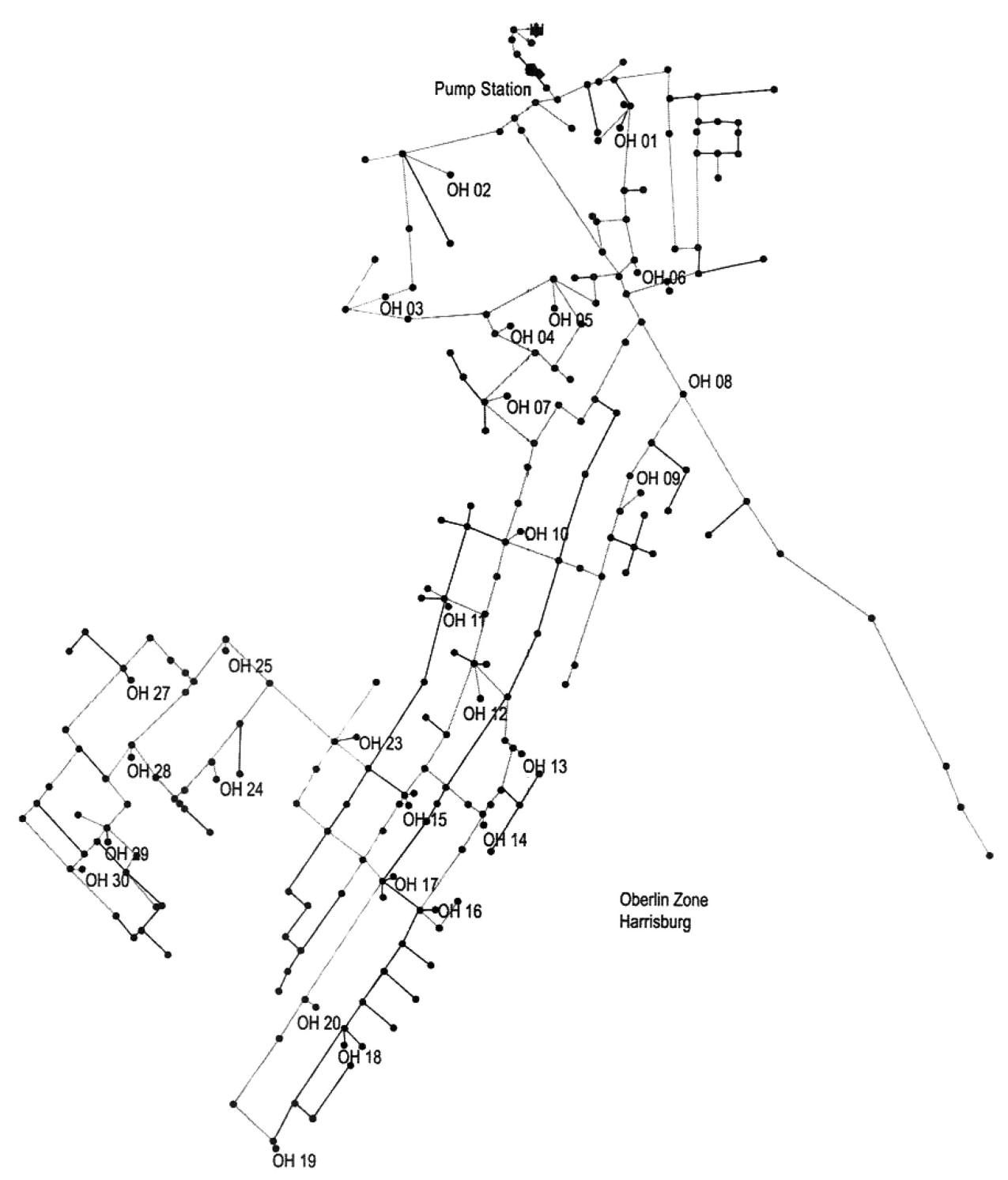

Figure 2: Oberlin zone of Harrisburg Water Distribution System.

into the system at the source (booster station in this case). Water quality at the outskirt of the system, however, is quite different from the nodes near the pump station. Figure 5 implies that significant chlorine decay has taken place from the source into the distribution system. Without good calibration on the water quality model, the field observed chlorine residual is away mismatched with the model simulated. Figures 3 and 5 demonstrate that the calibration 


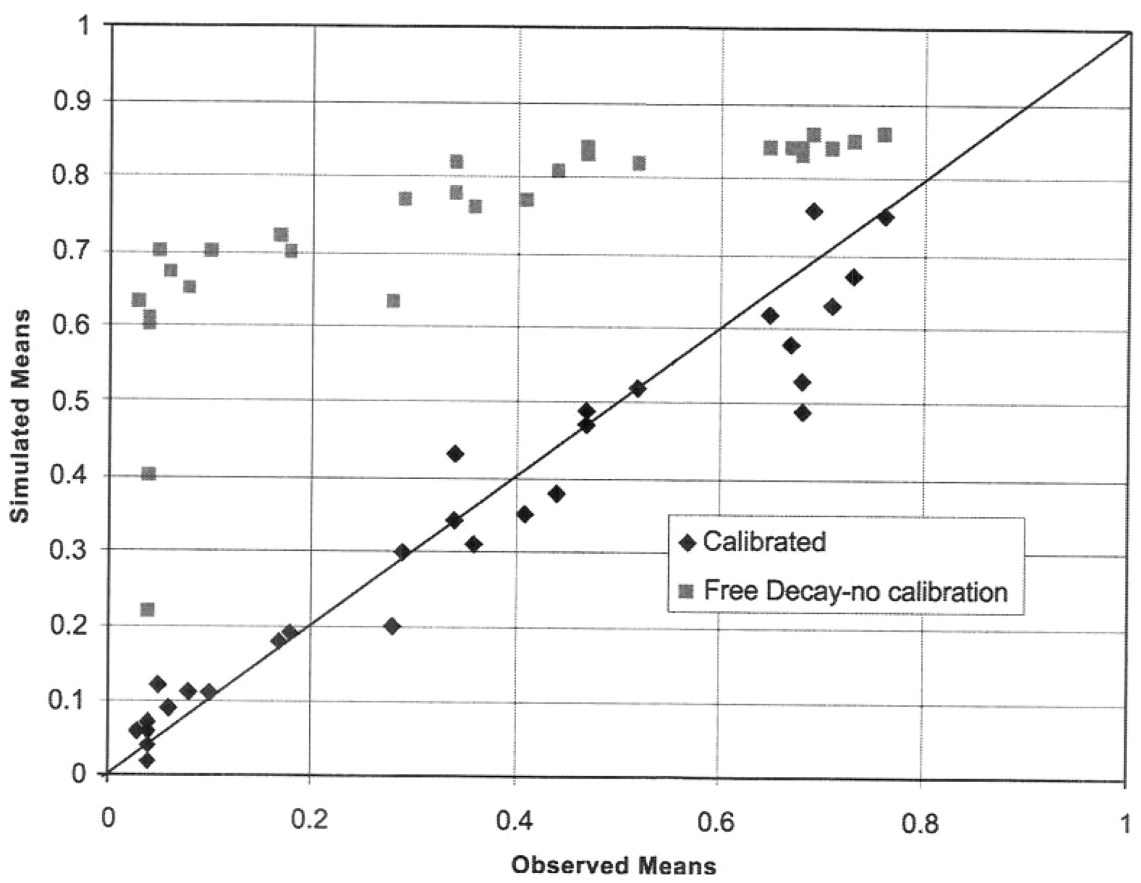

Figure 3: Comparison the observed and simulated mean of chlorine concentrations over 35 hours at 27 sampling locations.

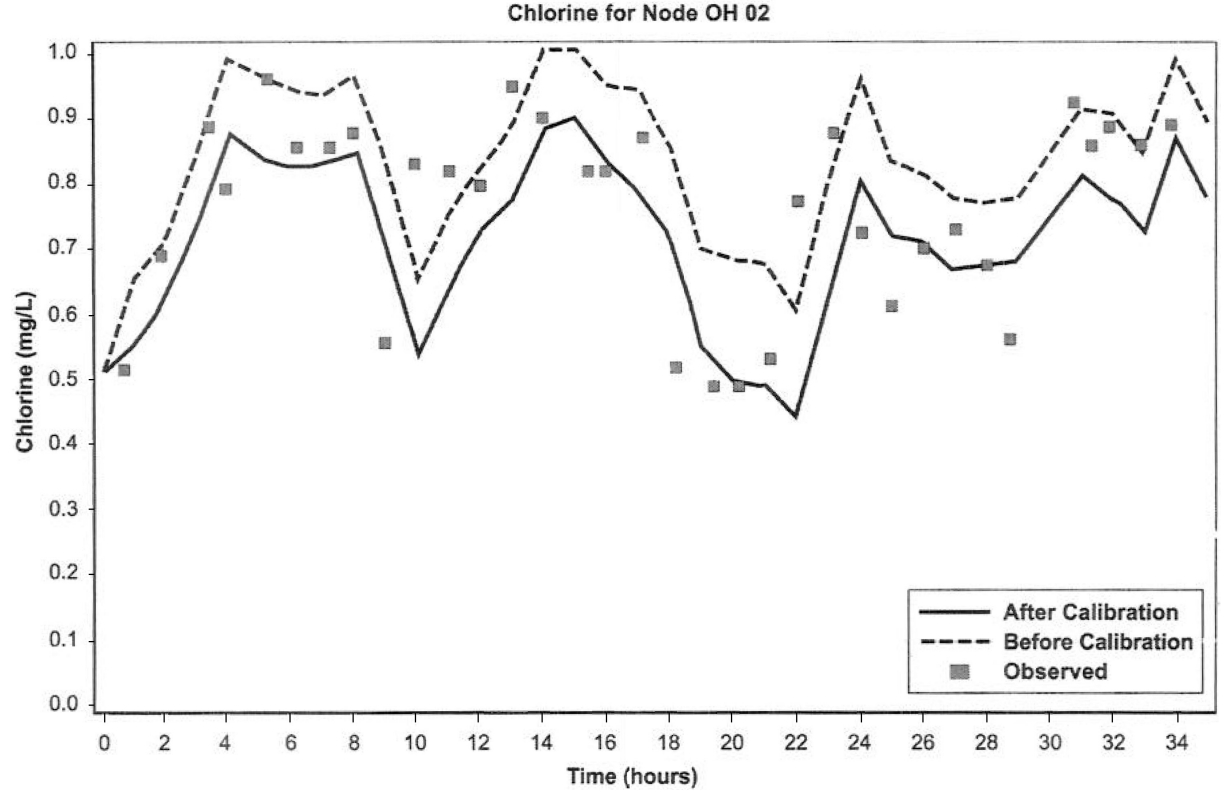

Figure 4: Comparison of the field observed and the model simulated chlorine concentration at node $\mathrm{OH} 02$ near the water source (booster station). 


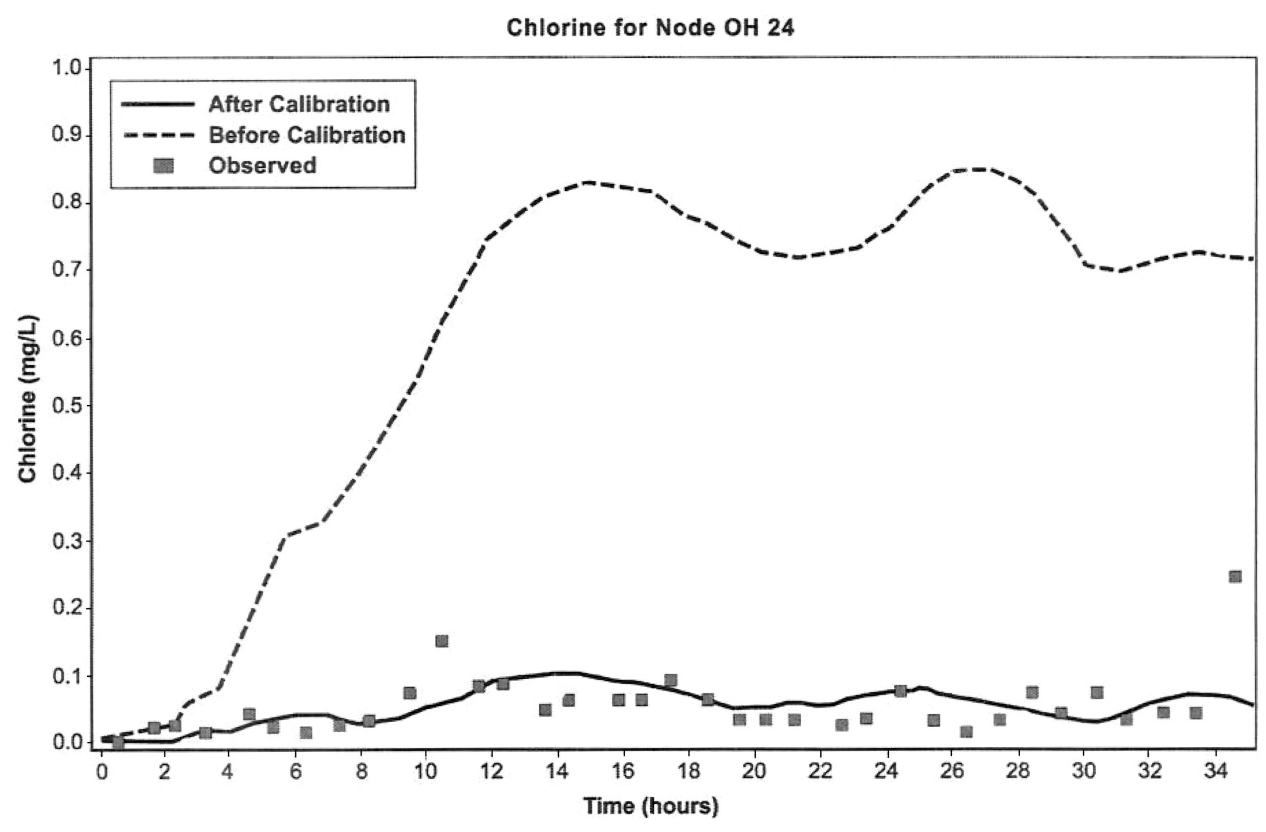

Figure 5: Comparison of the field observed and the model simulated chlorine concentration at node $\mathrm{OH} 24$ the outskirt of system.

approach successfully enhances the chlorine residual agreement, particularly for the nodes far apart from the source.

Three top calibration solutions are presented in Table 1 and compared with previous study. The best fitness of 1.309 with average difference of $0.045 \mathrm{mg} / \mathrm{L}$ has been achieved for 27 sampling stations over 35 hours while the previous study resulted in the fitness of 2.467 with average of $0.086 \mathrm{mg} / \mathrm{L}$. It clearly indicates that the better calibration solutions have been obtained by using the optimal calibration method. The solutions are ranked by the fitness value, the sum of absolute mean differences, which is resulted in by different correlation factor between pipe wall coefficient and roughness for pipe groups. The average of absolute mean differences is the same for all three solutions. It is no doubt that genetic algorithm calibration effectively improved the water quality model for this case study and a better solution has been obtained than the conventional approach. However, an accurate water quality model cannot be expected to be achieved by simply performing optimization calibration run. An insightful analysis must be undertaken for understanding the solution and the data points where the relatively greater discrepancies are resulted in. This is usually caused by poor data quality and abnormal model representation. Optimization modeling tool may help engineers quickly to reveal the weakest where good engineering judgment is applied to investigate the possible errors, 
14 Wu

Table 1: Comparison of optimal calibration solutions to previous study.

\begin{tabular}{|c|c|c|c|c|}
\hline Items & $\begin{array}{l}\text { Optimal } \\
\text { solution I }\end{array}$ & $\begin{array}{l}\text { Optimal } \\
\text { solution II }\end{array}$ & $\begin{array}{l}\text { Optimal } \\
\text { solution III }\end{array}$ & $\begin{array}{l}\text { Vasconcelos } \\
\text { et al. }{ }^{[12]}\end{array}$ \\
\hline $\begin{array}{l}\text { Fitness (sum of absolute mean } \\
\text { differences) }\end{array}$ & 1.3090 & 1.3106 & 1.3119 & 2.4670 \\
\hline $\begin{array}{l}\text { Average absolute mean } \\
\text { difference }(\mathrm{mg} / \mathrm{L})\end{array}$ & 0.0450 & 0.0450 & 0.0450 & 0.0860 \\
\hline $\begin{array}{l}\text { Bulk reaction order } \\
\text { Wall reaction order } \\
\text { Bulk }\end{array}$ & $\begin{array}{l}2 \\
0\end{array}$ & $\begin{array}{l}2 \\
0\end{array}$ & $\begin{array}{l}2 \\
0\end{array}$ & $\begin{array}{l}1 \\
0\end{array}$ \\
\hline $\begin{array}{l}\text { Pipe group } 1 \\
\text { Regction }\end{array}$ & $-1^{*}$ & -1 & -1 & -0.232 \\
\hline $\begin{array}{l}\text { Pipe group } 2 \\
\text { Coefficient }\end{array}$ & -1 & -1 & -1 & -0.232 \\
\hline $\begin{array}{l}\text { Pipe group } 3 \\
\text { Wall }\end{array}$ & -1 & -1 & -1 & -0.232 \\
\hline $\begin{array}{l}\text { Pipe group } 1 \\
\text { Correlation }\end{array}$ & -800 & -795 & -785 & -660 \\
\hline $\begin{array}{l}\text { Pipe group } 2 \\
\text { Coefficient }\end{array}$ & -470 & -470 & -470 & -660 \\
\hline Pipe group 3 & -600 & -600 & -600 & -660 \\
\hline
\end{tabular}

*Negative sign indicates chlorine decay (not growth).

which is an indispensable task to model calibration due to no perfect data exist for a real system.

\section{DISCUSSIONS}

The results of example water quality model calibration have shown the effectiveness of competent genetic algorithm for solving nonlinear constrained optimization problem. Conventionally, water quality model calibration is achieved by manually adjusting pipe wall reaction coefficient along with bottle test in a laboratory, which determines the bulk reaction order (either 0 or 1 ) and the bulk reaction rate. For the chlorine decay model, pipe wall reaction coefficient may be globally adjusted by using a correlation factor to roughness. It can simplify the complexity and reduces the work load for manual calibration. The previous study demonstrated that some good calibration results have been achieved for chlorine decay model of a relatively small system. However, it is unknown if the approach is applicable to a large real system with multiple water sources of mixed water quality. In addition, the correlation factor method only proved to be applicable to chlorine decay. For real water distribution system, water quality can be maintained by using other chemicals, pipe wall reaction rate may not be the same for all pipes, the bulk reaction can be in any order (not just 0 or 1 ) and also bulk reaction coefficient varies from location to location due to mixed water quality from different water sources. Thus the simplified approach of just calibrating pipe wall coefficient by a 
correlation factor is unlikely be able to handle all the complexity of a real water quality model. In contrast, the water quality calibration approach developed in this paper is generic and flexible method taking into account combination of different water quality parameters. It is able to consider the elementdependent reaction parameters (for both pipe and tank) and any reaction order. With the capability of grouping the pipes of similar characteristics, modeler is able to calibrate a water quality model of any constituent.

To achieve a good water quality calibration, a well calibrated EPS hydraulic model is essential before starting water quality calibration. The accuracy of water quality simulation relies on the hydraulic simulation results. A hydraulic simulation must be performed priori to a water quality analysis. It is the hydraulic simulation that provides the necessary flow and velocity information of each element to determine how a constituent is transported and reacted throughout a distribution system. This indicates that hydraulic calibration must be conducted before embarking on water quality model calibration, and also hydraulic model calibration must be carried out for extended period simulation. If there are errors in the hydraulic model, then forcing the water quality parameters to achieve calibration may result in a model that appears calibrated due to compensating errors.

\section{CONCLUSIONS}

Water quality modeling is an important means of providing system-wide information on water quality for evaluating routine system operation policy, thus maintaining and improving water quality throughout a system. Calibrating such a model ensures that a water quality model predicts what is happening in a real system. The approach presented in this paper has provided a generic tool and methodology for calibrating a water quality model of any constituent. It relieves modeler from trial and error process and thus enables engineers to construct an accurate model for effectively managing water quality in distribution systems to comply the public health.

\section{ACKNOWLEDGMENT}

Author would like to thank Dr. Walter Grayman and Dr. Lewis Rossman for providing the data used for this research.

\section{REFERENCES}

1. Chun, D.G.; Selznick, H.L. Computer modeling of distribution system water quality. Proc., Comp. Appl. Water Resour., ASCE, New York, 1985; 448-456.

2. Males, R.M.; Clark, R.M.; Welhrman, P.J.; Gates, W.E. Algorithm for mixing problems in water systems. ASCE J. Hydraul. Eng. 1985, 111 (2), 206-219. 
16 Wu

424 3. Liou, C.P.; Kroon, J.R. Propagation and distribution of waterborn substances in 425 networks. Proc. AWWA, DSS, Minneapolis, 1986.

426 4. Clark, R.M.; Grayman, W.M.; Males, R.M. Contaminant propagation in distribution 427 systems. J. Environ. Eng. 1988, 114 (4), 929-943.

428 5. Islam, M.R.; Chaudhry, M.H.; Clark, R.M. Inverse modeling of chlorine concentra429 tion in pipe networks under dynamic conditions. ASCE J. Environ. Eng. 1997, 123, $430 \quad 1033-1040$.

431 6. Grayman, W.M.; Clark, R.M.; Males, R.M. Modleing distribution water quality: 432 dynamic approach. J. Water Resour. Plan. Mgmt. ASCE 1988, 114, 295-312.

433 7. Liou, C.P.; Kroon, J.R. Modeling the propagation of waterborne substances in 434 distribution networks. J. AWWA 1987, 79 (11), 54-58.

435 8. Rossman, L.A.; Clark, R.M.; Grayman, W.M. Modeling chlorine residual in drinking 436 water distribution system. J. Environ. Engr. ASCE 1994, 120, 803-820.

437 9. Rossman, L.A.; Boulos, P.F. Numerical methods for modeling water quality in 438 distribution systems: a comparison. J. Water Resour. Plan. Mgmt. ASCE 1996, 122, $439 \quad 137-146$.

440 10. Wu, Z.Y.; Walski, T.M.; Mankowski, R.; Herrin, G.; Gurrieri, R.; Tryby, M. 441 Calibrating water distribution models via genetic algorithms, proceedings AWWA 442 Information Management Technology Conference, Kansas City, MO, 2002.

443 11. Wu, Z.Y.; Arniella, E.F.; Gianellaand, E. Darwin calibrator- Improving project 444 productivity and model quality for large water systems. J. AWWA 2004, 96 (10), 27-34.

445 12. Vasconcelos, J.J.; Rossman, L.A.; Grayman, W.M.; Boulos, P.F.; Clark, R.M. 446 Kinetics of chlorine decay. J. AWWA 1997, 89, 7.

447 13. Wu, Z.Y.; Simpson, A.R. Competent genetic algorithm optimization of water 448 distribution systems. J. Comp. Civ. Engr. ASCE 2001, 15 (2), 89-101. 